\title{
Technology Revolution of HLA Typing
}

Minzhong Tang1, Jun Li2, Yulu Huang3, Yuming Zheng3 and Yonglin Cai2*

${ }^{1}$ College of Life Science and Bio-Engineering, Beijing University of Technology, Beijing, P.R. China

${ }^{2}$ Wuzhou Health System Key Laboratory for Nasopharyngeal Carcinoma Etiology and Molecular Mechanism, Wuzhou Red Cross Hospital, Guangxi, P.R. China

${ }^{3}$ Wuzhou Cancer Research Institute, Guangxi, P.R. China

*Corresponding author: Tang M, Wuzhou Red Cross Hospital, Guangxi, 543002 P.R. China, E-mail: gxtom@163.com

Rec date: Apr 29, 2014; Acc date: May 27, 2014; Pub date: June 4, 2014

Copyright: ${ }^{\odot} 2014$ Tang M, et al. This is an open-access article distributed under the terms of the Creative Commons Attribution License, which permits unrestricted use, distribution, and reproduction in any medium, provided the original author and source are credited.

\section{Introduction}

The human leukocyte antigen (HLA) region is the most polymorphic in the human genome, which located on the chromosome $6 \mathrm{p} 21$ encodes more than 200 genes. HLA loci are known to influence susceptibility to numerous diseases including complex autoimmune and inflammatory diseases such as rheumatoid arthritis, ankylosing spondilitis, type 1 diabetes, and multiple sclerosis; nasopharyngeal cancer, Hodgkin disease and other cancers; infectious diseases including malaria, tuberculosis, and AIDS; rejection of hematopoietic stem cell or organ transplants [1-4].

The determination of the classical HLA alleles has been developed rapidly over past thirty years, especial in DNA typing techniques. The various typing method have been used for HLA DNA typing, such as incorporating restriction fragment length polymorphisms (RFLP) [5], sequence specific oligonucleotides hybridization (SSOs) [6], sequence specific primers (SSPs) [7] and capillary sequencing with chaintermination reaction (Sanger sequencing or SBT) [8]. More recently, next-generation sequencing (NGS) technologies, which are also referred as second-generation sequence technologies are new developments. NGS was initially introduced for whole genome sequencing and for quantitation of viral variants or genetic mutations in tumor tissues [9]. The most significant feature of NGS technologies is that a large amount of clonal sequences could be generated in a single sequencing run. To make NGS technology available for small and intermediate sized laboratories, the major vendors of second generation sequencing technology have released their benchtop sequencer. Those benchtop NGS platforms offer sufficient throughput for a variety of targeted sequencing applications and fit easily into a medium sized equipment budget plan. The significant improvement in NGS make it has great potential to overcome traditional sequencingbased typing, resulted in revolutionary changes in HLA typing. Indeed, three main platforms, the Roche 454 GS Junior, the Illumina Miseq and the Ion Torrent system, using NGS have been developed recently, and some platforms are still to come.

Here we evaluate the application of these new sequencing platforms in HLA and summarize of the technical specifications of each of these instruments.

The Roche 454 system was the many firsts NGS system that had been introduced into market. Bentley et al. demonstrated the highresolution HLA genotyping by amplicon pooling approach in Roche 454/FLX sequencer in 2009 [10]. Nowadays, commercially as the GS GType HLA Primer Sets, enable high- and medium-resolution genotyping of the class I and II loci of the HLA genes running on both Roche 454GS FLX and GS Junior systems. 454 sequencing system employs emulsion polymerase chain reaction, barcoding of amplicons and long range sequence reads that allow for setting phase for linked polymorphisms within the sequenced DNA library fragment, perfectly fitting to the highly polymorphic immunogenetics diagnosed. Methodically, titanium libraries of single-stranded template DNA fragments were prepared for emPCR and sequencing using a GS FLX Titanium Rapid Library Preparation kit. The multiplexed libraries were clonally amplified by emPCR in order to produce hundreds of thousands or millions of copies of the same template sequence so that sufficient signal would be generated to be easily detected and recorded by the sequencing system. Currently, the Roche GS GType HLA Primer Sets 454 Life Sciences for the 454 FLX and GS Junior platform is the only commercially available HLA typing kit for next-generation sequencing. Amplicon-based sequencing results generated by 454 systems could be imported to third party genotyping software, Conexio ATF software (Conexio Genomics, Fremantle, Australia), with a user-friendly interface for analysis [11]. These software developments have improved the robustness and reliability and proved with excellent results in a study with eight different reference laboratories [12]. The disadvantage of 454 NGS platform was 454 technology demands expensive reagents. The inherently high costs drive the manufacturer to seek for new technologies.

In recent years, the sequencing industry has been dominated by Illumina. Illumina released the Miseq NGS system in early 2011, implementing the Solexa Technology, initially developed at the University of Cambridge. Methodically, after libraries were denatured and diluted, DNA molecules are bound to a solid phase, so call flow cell, via forward and reverse adaptor oligonucleotides. Complementary "adaptor" specific sequences are attached to the templates during library generation at their 5'and 3'ends, thus enabling them to bind to the flow cell surface. To enhance the sequencing signal, single DNA molecules are bound to the surface of the flow cell, and then bridgeamplified to form clusters resulting in up to 1000 densely packed identical copies of the original solid phase-bound template. Clusters are imaged using LED and filter combinations specific to each of the four fluorescently labeled dideoxynucleotides. After imaging of one tile of the flow cell is complete, the flow cell is moved into place to expose the next tile. The process is repeated for each cycle of sequencing. The data are collected for each cluster separately and used to generate the 'paired-end output files'. In Miseq's original form, the read lengths were limited to a maximum of $2 \times 150 \mathrm{bp}$. However, Illumina improves their sequencing chemistry and sequencing data collection continuously. In the latest version of Miseq (V3), read lengths reached up to $2 \times 300 \mathrm{bp}$ paired-end. The long read lengths improvement allows Miseq well applicable for HLA typing. Basically two different principles may be applied. In so-called 'amplicon-based' approaches, sequencing templates are generated by specific PCR of the genomic regions of interest, followed by subsequent pooling and sequencing of these templates. In 'shotgun' approaches, genomic DNA or long range 
PCR products are randomly fragmented followed by enzymatical endrepair and adapter/index oligonucleotide addition steps [13].

Ion Torrent launched in February 2010 with a sequencer based not on dye-labeled oligonucleotides and expensive optics but on ion detection [14]. The Ion Personal Genome Machine (PGM) produces the fastest results of any benchtop next-generation sequencer, offering a one-day workflow for most applications. Briefly, DNA fragments with specific adapter sequences are linked to and then clonally amplified by emulsion PCR on the surface of 3-micron diameter beads, known as Ion Sphere Particles; The templated beads are loaded into proton- sensing wells that are fabricated on a silicon wafer and sequencing is primed from a specific location in the adapter sequence; As sequencing proceeds, each of the four bases is introduced sequentially; The polymerase incorporates the appropriate oligonucleotide into the growing strand, and the hydrogen ion that is released changes the $\mathrm{pH}$ in the solution, which is detected by an ion sensor. This allows sequencing in real time. Ion Torrent platform has rapidest and scalable sequencing comparing with other NGS platforms. A 200 bps run only takes $2 \mathrm{~h}$ for completion and the equipment needed for signal detection is comparatively inexpensive. The disadvantages concern the uneven coverage and the high manual workload, and the sequencing error rate is higher than those of other platforms [15].

High-throughput HLA genotyping using NGS have been reported by several groups [12,16-20]. Most of these studies amplified in an exon based strategy and sequenced in a multiplexed manner. The super high-resolution technologies give the great advantage to exploit disease associations of HLA in detail. For example, whole exome sequencing of HLA allow researches perform amino acid variation analyses precisely. A novel method for analysis of $\mathrm{MHC} /$ disease associations that additionally incorporates structural and functional information about the HLA molecules to help illuminate the biological nature of disease associations based on variations in the functional sequence features to augment allele-based association analyses had been reported [21]. A genetic study from our group had demonstrated that a powerful genetic influence on NPC onset, implicates explicit HLA alleles, peptide recognition motifs, and amino acid variants that confer strong genetic influence on the development of NPC [22].

New technologies substantially improve the allele resolution for genotyping and lead to a better understanding of HLA disease association. For requirements of clinical testing and compliance with regulatory issues, in addition to the specifications and performance of the sequencer, extensive development and validation are necessary for HLA-typing applications.

\section{Acknowledgement}

This project has been funded in whole or in part with Guangxi science and technology grant, China (114003A-49); Guangxi nature science foundation grant, China (2013GXNSFCA019014) and Wuzhou science and technology grant, China (201301046).

\section{References}

1. Cotton LA, Kuang XT, Le AQ, Carlson JM, Chan B, et al. (2014) Genotypic and functional impact of HIV-1 adaptation to its host population during the North American epidemic. PLoS Genet 10: e1004295.
2. Tang M, Zeng Y, Poisson A, Marti D, Guan L, et al. (2010) Haplotypedependent HLA susceptibility to nasopharyngeal carcinoma in a Southern Chinese population. Genes Immun 11: 334-342.

3. Zecca M, Strocchio L, Pagliara D, Comoli P, Bertaina A, et al. (2014) HLA-haploidentical $\mathrm{T}$ cell-depleted allogeneic hematopoietic stem cell transplantation in children with Fanconi anemia. Biol Blood Marrow Transplant 20: 571-576.

4. Jin H, Arase N, Hirayasu K, Kohyama M, Suenaga T, et al. (2014) Autoantibodies to IgG/HLA class II complexes are associated with rheumatoid arthritis susceptibility. Proc Natl Acad Sci U S A 111: 3787-3792.

5. Ota M, Fukushima H, Kulski JK, Inoko H (2007) Single nucleotide polymorphism detection by polymerase chain reaction-restriction fragment length polymorphism. Nat Protoc 2: 2857-2864.

6. Saiki RK, Walsh PS, Levenson CH, Erlich HA (1989) Genetic analysis of amplified DNA with immobilized sequence-specific oligonucleotide probes. Proc Natl Acad Sci U S A 86: 6230-6234.

7. Olerup O, Zetterquist H (1992) HLA-DR typing by PCR amplification with sequence-specific primers (PCR-SSP) in 2 hours: an alternative to serological DR typing in clinical practice including donor-recipient matching in cadaveric transplantation. Tissue Antigens 39: 225-235.

8. Santamaria P, Lindstrom AL, Boyce-Jacino MT, Myster SH, Barbosa JJ, et al. (1993) HLA class I sequence-based typing. Hum Immunol 37: 39-50.

9. Abel HJ, Duncavage EJ (2013) Detection of structural DNA variation from next generation sequencing data: a review of informatic approaches. Cancer Genet 206: 432-440.

10. Bentley G, Higuchi R, Hoglund B, Goodridge D, Sayer D, et al. (2009) High-resolution, high-throughput HLA genotyping by next-generation sequencing. Tissue Antigens 74: 393-403.

11. Moonsamy PV, Williams T, Bonella P, Holcomb CL, Höglund BN, et al. (2013) High throughput HLA genotyping using 454 sequencing and the Fluidigm Access Arrayâ,„ $₫$ System for simplified amplicon library preparation. Tissue Antigens 81: 141-149.

12. Holcomb CL, Höglund B, Anderson MW, Blake LA, Böhme I, et al. (2011) A multi-site study using high-resolution HLA genotyping by next generation sequencing. Tissue Antigens 77: 206-217.

13. Lange V, Böhme I, Hofmann J, Lang K, Sauter J, et al. (2014) Costefficient high-throughput HLA typing by MiSeq amplicon sequencing. BMC Genomics 15: 63.

14. Rothberg JM, Hinz W, Rearick TM, Schultz J, Mileski W, et al. (2011) An integrated semiconductor device enabling non-optical genome sequencing. Nature 475: 348-352.

15. Ross MG, Russ C, Costello M, Hollinger A, Lennon NJ, et al. (2013) Characterizing and measuring bias in sequence data. Genome Biol 14: R51.

16. Gabriel C, Danzer M, Hackl C, Kopal G, Hufnagl P, et al. (2009) Rapid high-throughput human leukocyte antigen typing by massively parallel pyrosequencing for high-resolution allele identification. Hum Immunol 70: 960-964.

17. Lind C, Ferriola D, Mackiewicz K, Heron S, Rogers M, et al. (2010) Nextgeneration sequencing: the solution for high-resolution, unambiguous human leukocyte antigen typing. Hum Immunol 71: 1033-1042.

18. Shiina T, Suzuki S, Ozaki Y, Taira H, Kikkawa E, et al. (2012) Super high resolution for single molecule-sequence-based typing of classical HLA loci at the 8-digit level using next generation sequencers. Tissue Antigens 80: 305-316.

19. Wang C, Krishnakumar S, Wilhelmy J, Babrzadeh F, Stepanyan L, et al. (2012) High-throughput, high-fidelity HLA genotyping with deep sequencing. Proc Natl Acad Sci U S A 109: 8676-8681.

20. Erlich RL, Jia X, Anderson S, Banks E, Gao X, et al. (2011) Nextgeneration sequencing for HLA typing of class I loci. BMC Genomics 12: 42.

21. Karp DR, Marthandan N, Marsh SG, Ahn C, Arnett FC, et al. (2010) Novel sequence feature variant type analysis of the HLA genetic association in systemic sclerosis. Hum Mol Genet 19: 707-719. 
Citation: $\quad$ Tang M, Li J, Huang Y, Zheng Y and Cai Y (2014) Technology Revolution of HLA Typing. Next Generat Sequenc \& Applic 1: 104. doi: 10.4172/2469-9853.1000104

Page 3 of 3

22. Tang M, Lautenberger JA, Gao X, Sezgin E, Hendrickson SL, et al. (2012) The principal genetic determinants for nasopharyngeal carcinoma in
China involve the HLA class I antigen recognition groove. PLoS Genet 8: e1003103. 